TRANSACTIONS OF THE

AMERICAN MATHEMATICAL SOCIETY

Volume 353, Number 6, Pages 2441-2457

S 0002-9947(01)02721-0

Article electronically published on January 3, 2001

\title{
MODEL CATEGORY STRUCTURES ON CHAIN COMPLEXES OF SHEAVES
}

\author{
MARK HOVEY
}

\begin{abstract}
The unbounded derived category of a Grothendieck abelian category is the homotopy category of a Quillen model structure on the category of unbounded chain complexes, where the cofibrations are the injections. This folk theorem is apparently due to Joyal, and has been generalized recently by Beke. However, in most cases of interest, such as the category of sheaves on a ringed space or the category of quasi-coherent sheaves on a nice enough scheme, the abelian category in question also has a tensor product. The injective model structure is not well-suited to the tensor product. In this paper, we consider another method for constructing a model structure. We apply it to the category of sheaves on a well-behaved ringed space. The resulting flat model structure is compatible with the tensor product and all homomorphisms of ringed spaces.
\end{abstract}

\section{INTRODUCTION}

The unbounded derived category of an abelian category $\mathcal{A}$ is defined to be the quotient of the category of unbounded chain complexes $\operatorname{Ch}(\mathcal{A})$ obtained by adjoining formal inverses to all the quasi-isomorphisms (maps that induce homology isomorphisms). With this definition, one has little control over the morphisms in the derived category. In fact, it is not known in general that the derived category is a category, in the usual sense of having only a set of maps between any two objects.

One excellent way to cope with this problem is Quillen's theory of model categories Qui67. If one can prove that $\operatorname{Ch}(\mathcal{A})$ is a model category with the quasiisomorphisms as the weak equivalences, then it follows from Quillen's theory that the derived category is a category. Furthermore, one has a fairly simple description of the morphisms from $X$ to $Y$ in the derived category as chain homotopy classes of chain maps from a cofibrant replacement for $X$ to a fibrant replacement for $Y$.

It is a folk theorem, apparently due to Joyal [Joy84] (see Bek99]), that $\mathrm{Ch}(\mathcal{A})$ is a model category with quasi-isomorphisms as weak equivalences and monomorphisms as cofibrations whenever $\mathcal{A}$ is a Grothendieck abelian category. We call this the injective model structure. This applies in particular to the category of sheaves over a ringed space or topos, and to the category of quasi-coherent sheaves over a quasi-compact, quasi-separated scheme. This result has been rediscovered in various forms by several people (including the author). Moerdijk and Pronk constructed the injective model structure for sheaves in [MP92]. Many of the results of

Received by the editors February 24, 2000.

2000 Mathematics Subject Classification. Primary 18F20, 14F05, 18E15, 18E30, 18G35, $55 \mathrm{U} 35$. 
Spaltenstein on unbounded complexes [Spa88 and their generalizations in [TLS99] follow immediately from the existence of the injective model structure.

However, the categories just mentioned are closed symmetric monoidal under the tensor product, as are most Grothendieck categories used in practice. In this case, the category $\operatorname{Ch}(\mathcal{A})$ will also be closed symmetric monoidal, with the usual definitions of Hom and tensor of chain complexes. The injective model structure does not interact well with the tensor product, making it useless for defining or studying the derived tensor product.

We therefore discuss a different method for constructing a model structure on $\operatorname{Ch}(\mathcal{A})$. This method enables us to define a different model structure on $\operatorname{Ch}(\mathcal{A})$ in case $\mathcal{A}$ has a set of generators of finite projective dimension. In particular, we apply it when $\mathcal{A}$ is the category of quasi-coherent sheaves on a nice enough scheme, using the locally free sheaves as the generators. Though the resulting locally free model structure is still not compatible with the tensor product, it does give us some information about the resulting derived category that does not seem accessible from the injective model structure.

But this method works better when $\mathcal{A}$ is the category of sheaves on a ringed space $(S, \mathcal{O})$ satisfying a hypothesis related to finite cohomological dimension. In this case, we construct a flat model structure on $\operatorname{Ch}(\mathcal{A})$ that is compatible with the tensor product. We then get model categories of differential graded $\mathcal{O}$-algebras and of differential graded modules over a given differential graded $\mathcal{O}$-algebra. The flat model structure is also natural for arbitrary maps of ringed spaces.

To understand this paper, the reader needs to know some basic facts about model categories, Grothendieck categories, and sheaves. A good introduction to model categories is [DS95]. The book [Hov98] is a more in-depth study, but still starting from scratch. All the terms we need are defined in [Hov98]; we will give specific references as needed. For Grothendieck categories, [Ste75] is a basic reference. We also need the fact that every object $X$ in a Grothendieck category $\mathcal{A}$ is small, in the sense that $\mathcal{A}(X,-)$ commutes with $\kappa$-indexed colimits, for all cardinals $\kappa$ with sufficiently large cofinality. This follows from the important fact that Grothendieck categories are locally presentable [Bek99, Proposition 3.10]. Because we have been unable to find a published reference for this fact, we include a direct proof of smallness in an appendix, based on the Gabriel-Popescu theorem. For sheaves and schemes, we try to refer mostly to [Har77], but we occasionally need more advanced results.

The author would like to thank Matthew Ando and Amnon Neeman for helpful discussions about sheaves, and Dan Christensen and the referee for useful suggestions.

\section{A METHOD FOR BUILDING MODEL STRUCTURES}

In this section, we attempt to build a model structure on $\operatorname{Ch}(\mathcal{A})$ for a Grothendieck category $\mathcal{A}$ by generalizing the usual projective model structure when $\mathcal{A}=R$-Mod for some ring $R$. Our approach is related to, but not identical with, the method of [Chr98]; the difference is that we need the weak equivalences to be the quasi-isomorphisms, whereas Christensen is willing to relax that hypothesis.

Recall from [Hov98, Section 2.3] that the projective model structure on $\operatorname{Ch}(\mathcal{A})$, where $\mathcal{A}=R$-Mod for some ring $R$, is a cofibrantly generated model structure (see Hov98, Section 2.1] for the definition of this term), with generating cofibrations 
$I=\left\{S^{n-1} R \rightarrow D^{n} R\right\}$ and generating trivial cofibrations $J=\left\{0 \rightarrow D^{n} R\right\}$. Here $n$ runs through all integers, $S^{n-1} M$ is the complex whose only nonzero object is $M$ in dimension $n-1$, and $D^{n} M$ is the complex whose only nonzero objects are $M$ in dimensions $n$ and $n-1$. Our plan is to replace the map $0 \rightarrow R$ by a set of monomorphisms $\mathcal{M}$.

Recall that, given a collection of maps $J, J$-inj is the class of all maps $p$ having the right lifting property with respect to $J$ [Hov98, Section 2.1.2]; this means that, given a commutative square

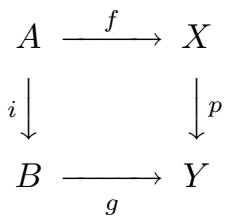

where $i \in J$, there is a map $h: B \rightarrow X$ such that $h i=f$ and $p h=g$. Similarly, $J$-proj is the class of maps having the left lifing property with respect to $J$. We let $J$-cof $=(J$-inj)-proj, and $J$-fib $=(J$-proj)-inj.

In a Grothendieck category $\mathcal{A}, J$-cof is the smallest class of maps containing $J$ and closed under retracts (in the category of maps), pushouts, and transfinite compositions. This follows from the small object argument (see Hov98. Corollary 2.1.15]), using the fact, proved in the Appendix, that every object in a Grothendieck category is small.

Definition 1.1. Suppose $\mathcal{M}$ is a set of monomorphisms in a Grothendieck category $\mathcal{A}$. Let $\mathcal{F}$ denote the set of codomains of the maps of $\mathcal{M}$. We will say that $\mathcal{M}$ is pointed if $0 \in \mathcal{F}$ and, if $F \in \mathcal{F}$, then $0 \rightarrow F$ is in $\mathcal{M}$. Define $J$ to be the set of all $D^{n} f$, where $n$ is an integer and $f \in \mathcal{M}$. Define $I$ to be the union of $J$ and the maps $S^{n-1} F \rightarrow D^{n} F$ for $F \in \mathcal{F}$ and $n$ an integer. Then define a map $p$ to be an $\mathcal{M}$-fibration if $p$ is in $J$-inj, and define $p$ to be an $\mathcal{M}$-cofibration if $p$ is in $I$-cof.

If $\mathcal{M}$ consists only of the maps $0 \rightarrow F$ for $F \in \mathcal{F}$, then we recover the definitions of [Chr98. However, the results we prove in this section do not recover the results of Chr98, as we always use quasi-isomorphisms as our weak equivalences and thus require more hypotheses.

Our goal is to determine conditions on $\mathcal{M}$ under which the quasi-isomorphisms, the $\mathcal{M}$-cofibrations, and the $\mathcal{M}$-fibrations determine a model structure on $\operatorname{Ch}(\mathcal{A})$. We use the recognition theorem [Hov98, Theorem 2.1.19]. Since the maps of $J$ are injective quasi-isomorphisms in $I$-cof, the maps of $J$-cof will also be. Indeed, it suffices to show that injective quasi-isomorphisms are closed under retracts (clear), pushouts, and transfinite compositions. For pushouts, use the fundamental theorem of homological algebra. For transfinite compositions, use the fact that homology commutes with colimits in any AB5 category. To apply the recognition theorem, then, we need to show that the maps of $I$-inj coincide with the maps that are both $\mathcal{M}$-fibrations and quasi-isomorphisms.

We begin by characterizing the $\mathcal{M}$-fibrations.

Definition 1.2. Suppose $\mathcal{M}$ is a pointed set of monomorphisms in a Grothendieck category $\mathcal{A}$. Define an object $X$ of $\mathcal{A}$ to be $\mathcal{M}$-flasque if $\mathcal{A}(f, X)$ is surjective for all $f \in \mathcal{M}$.

This definition is a generalization of the usual notion of flasque, or flabby, sheaves. We will discuss this in detail in the next section. 
Let us denote the category of chain complexes of abelian groups by $\mathrm{Ch}(\mathbb{Z})$.

Proposition 1.3. Suppose $\mathcal{M}$ is a pointed set of monomorphisms in a Grothendieck category $\mathcal{A}$. Then a map $p: X \rightarrow Y$ in $\operatorname{Ch}(\mathcal{A})$ is an $\mathcal{M}$-fibration if and only if $\mathcal{A}(F, p)$ is a surjection in $\mathrm{Ch}(\mathbb{Z})$ for all $F$ in $\mathcal{F}$ and $\operatorname{ker} p$ is dimensionwise $\mathcal{M}$-flasque.

In particular, if $\mathcal{F}$ is a set of generators for $\mathcal{A}$, then $\mathcal{M}$-fibrations are surjective. To see this, consider the map from $Y_{n}$ into the cokernel of $p_{n}$.

Proof. Adjointness implies that $p$ has the right lifting property with respect to $D^{n} B \stackrel{D^{n} f}{\longrightarrow} D^{n} C$ if and only if the map

$$
\mathcal{A}\left(C, X_{n}\right) \rightarrow \mathcal{A}\left(C, Y_{n}\right) \times_{\mathcal{A}\left(B, Y_{n}\right)} \mathcal{A}\left(B, X_{n}\right)
$$

is surjective. Applying this when $f$ is the map $0 \rightarrow F$ for $F \in \mathcal{F}$, we find that, if $p$ is an $\mathcal{M}$-fibration, then $\mathcal{A}(F, p)$ is surjective. Furthermore, if $p$ is an $\mathcal{M}$-fibration, then $\operatorname{ker} p \rightarrow 0$ is in $J$-inj. Applying the above criterion, we find that $\operatorname{ker} p$ is dimensionwise $\mathcal{M}$-flasque.

Conversely, suppose $\mathcal{A}(F, p)$ is a surjection for all $F \in \mathcal{F}$ and $K=\operatorname{ker} p$ is dimensionwise $\mathcal{M}$-flasque. Suppose $f: B \rightarrow C$ is in $\mathcal{M}$. We have an exact sequence

$$
0 \rightarrow \mathcal{A}\left(B, K_{n}\right) \rightarrow \mathcal{A}\left(B, X_{n}\right) \rightarrow \mathcal{A}\left(B, Y_{n}\right)
$$

and a similar exact sequence that is in fact short exact when $B$ is replaced by $C$. By pulling back the exact sequence for $B$ through the map $\mathcal{A}\left(f, Y_{n}\right)$, we obtain the following commutative diagram.

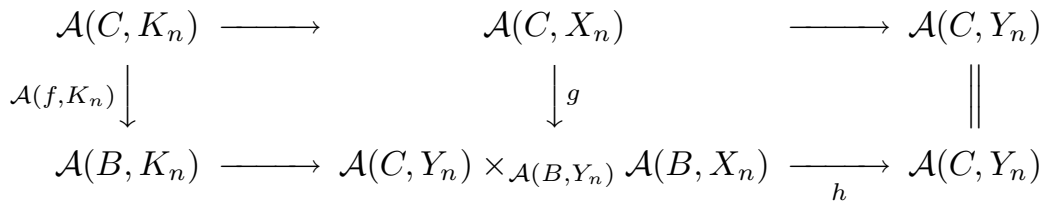

Note that, in this diagram, the composite $h g$ is surjective, so $h$ is surjective. Therefore we have a map of short exact sequences. Since $K$ is dimensionwise $\mathcal{M}$-flasque, the map $\mathcal{A}\left(f, K_{n}\right)$ is surjective. The snake lemma now implies that $g$ is surjective, so $p$ is an $\mathcal{M}$-fibration.

Proposition 1.4. Suppose $\mathcal{M}$ is a pointed set of monomorphisms in a Grothendieck category $\mathcal{A}$. Suppose in addition that the set $\mathcal{F}$ of codomains of $\mathcal{M}$ generates $\mathcal{A}$. Then every map of complexes $p: X \rightarrow Y$ in $I$-inj is both an $\mathcal{M}$-fibration and a quasi-isomorphism.

Proof. Recall that the functor $S^{n-1}: \mathcal{A} \rightarrow \mathrm{Ch}(\mathcal{A})$ is left adjoint to the functor that takes $X$ to $Z_{n-1} X$, the cycles in $X_{n-1}$. This implies that $p$ is in $I$-inj if and only if it is an $\mathcal{M}$-fibration and the map

$$
\mathcal{A}\left(F, X_{n}\right) \rightarrow \mathcal{A}\left(F, Y_{n}\right) \times_{\mathcal{A}\left(F, Z_{n-1} Y\right)} \mathcal{A}\left(F, Z_{n-1} X\right)
$$

is surjective for all $n$ and $F \in \mathcal{F}$. Let $K=\operatorname{ker} p$. If $p \in I$-inj, then the map $K \rightarrow 0$ is as well. Hence the map $\mathcal{A}\left(F, K_{n}\right) \rightarrow \mathcal{A}\left(F, Z_{n-1} K\right)$ is surjective for all $n$ and all $F \in \mathcal{F}$. Since $\mathcal{F}$ is a set of generators for $\mathcal{A}$, this implies that the map $K_{n} \rightarrow Z_{n-1} K$ is surjective, and hence that $K$ has no homology. A similar argument shows that $p$ is surjective, and so the long exact sequence implies that $p$ is a quasi-isomorphism. 
If $\mathcal{F}$ is not a generating set for $\mathcal{A}$, we can still say that, if $p \in I$-inj, then $\mathcal{A}(F, p)$ is a surjective quasi-isomorphism for all $F \in \mathcal{F}$.

To complete the construction of our model structure, we need to know that every map that is both an $\mathcal{M}$-fibration and a quasi-isomorphism is in $I$-inj. We begin with a lemma.

Lemma 1.5. Suppose $\mathcal{M}$ is a pointed set of monomorphisms in a Grothendieck category $\mathcal{A}$, and let $\mathcal{F}$ be the set of codomains of $\mathcal{M}$. Suppose $p: X \rightarrow Y$ is a map in $\operatorname{Ch}(\mathcal{A})$ such that $\mathcal{A}(F, p)$ is surjective for all $F \in \mathcal{F}$. Then $p$ is in $I$-inj if and only if $\operatorname{ker} p \rightarrow 0$ is in $I$-inj.

Proof. The only if implication is clear. Suppose $\mathcal{A}(F, p)$ is surjective for all $F \in \mathcal{F}$, and let $K=\operatorname{ker} p$. Suppose $K \rightarrow 0$ is in $I$-inj. In particular, this means that $K$ is dimensionwise $\mathcal{M}$-flasque, so $p \in J$-inj. In order to show that $p$ is in $I$-inj, we must show that, given $F \in \mathcal{F}$, a map $x: F \rightarrow Z_{n-1} X$, and a map $y: F \rightarrow Y_{n}$ such that $d \circ y=p \circ x$, there is a map $x^{\prime}: F \rightarrow X_{n}$ such that $p \circ x^{\prime}=y$ and $d \circ x^{\prime}=x$. First choose $z: F \rightarrow X_{n}$ such that $p \circ z=y$, using the fact that $\mathcal{A}(F, p)$ is surjective. Then $p \circ(d \circ z-x)=0$, so $d z-x: F \rightarrow Z_{n-1} K$. Since $K \rightarrow 0$ is in $I$-inj, there is a map $w: F \rightarrow K_{n}$ such that $d \circ w=d \circ z-x$. Now let $x^{\prime}=z-w$.

Proposition 1.6. Suppose $\mathcal{M}$ is a pointed set of monomorphisms in a Grothendieck category $\mathcal{A}$. Suppose that the set $\mathcal{F}$ of codomains of $\mathcal{M}$ generates $\mathcal{A}$, and, furthermore, suppose that if $K$ is an acyclic, dimensionwise $\mathcal{M}$-flasque complex and $F \in \mathcal{F}$, then $\mathcal{A}(F, K)$ is an acyclic complex of abelian groups. Then, if $p: X \rightarrow Y$ is an $\mathcal{M}$-fibration and quasi-isomorphism in $\mathrm{Ch}(\mathcal{A})$, then $p$ is in I-inj.

Proof. By Lemma 1.5 it suffices to show that $K=\operatorname{ker} p \rightarrow 0$ is in $I$-inj. But $K$ is an acyclic dimensionwise flasque complex, and so $\mathcal{A}(F, K)$ is also acyclic. Hence the map $\mathcal{A}\left(F, K_{n}\right) \rightarrow \mathcal{A}\left(F, Z_{n-1} K\right)$ is surjective, and so $K \rightarrow 0$ is in $I$-inj.

We have proved the following theorem.

Theorem 1.7. Suppose $\mathcal{M}$ is a pointed set of monomorphisms in a Grothendieck category $\mathcal{A}$ such that the set of codomains $\mathcal{F}$ of $\mathcal{M}$ forms a generating set of $\mathcal{A}$ and, for all acyclic, dimensionwise $\mathcal{M}$-flasque complexes $X$ and for all $F \in \mathcal{F}$, the complex $\mathcal{A}(F, X)$ is acyclic. Then $\operatorname{Ch}(\mathcal{A})$ is a proper cofibrantly generated model category, where the weak equivalences are the quasi-isomorphisms, the fibrations are the $\mathcal{M}$-fibrations, and the cofibrations are the $\mathcal{M}$-cofibrations.

We comment on the word "proper" in the above theorem. A model category is called left proper if the pushout of a weak equivalence through a cofibration is again a weak equivalence; right proper is defined dually. A model category is proper if it is both left and right proper. The long exact sequence in homology implies that any model structure on $\operatorname{Ch}(\mathcal{A})$ will be proper as long as weak equivalences coincide with quasi-isomorphisms, every cofibration is an injection, and every fibration is a surjection.

One interesting feature of the hypotheses of this theorem is that, if they are true for a given set of monomorphisms $\mathcal{M}$ with codomains $\mathcal{F}$, then they remain true if we expand $\mathcal{M}$ by adding any set of monomorphisms whose codomains are all in $\mathcal{F}$. So in fact we get many different model structures with the same weak equivalences, all relying on more or less stringent definitions of "flasque".

One might hope that we would still get a model structure on $\operatorname{Ch}(\mathcal{A})$ if we drop all hypotheses about the set of monomorphisms $\mathcal{M}$. The weak equivalences would 
have to change, probably to maps $f$ such that $\mathcal{A}(F, f)$ is a quasi-isomorphism for all $F \in \mathcal{F}$. With this definition, an appropriately modified version of Proposition 1.6 does hold. However, we do not know if the maps of $J$-cof are weak equivalences with this definition.

\section{Generators of Finite projective Dimension}

In this section, we apply the method of the previous section to construct a new model structure on $\operatorname{Ch}(\mathcal{A})$, when $\mathcal{A}$ is a Grothendieck category with generators of finite projective dimension. We apply this construction to the category of quasicoherent sheaves over a nice enough scheme.

Recall that an object $B$ is said to have finite projective dimension if there is an integer $n_{0}$ such that $\operatorname{Ext}_{\mathcal{A}}^{n}(B, C)=0$ for all $n \geq n_{0}$ and all objects $C$ of $\mathcal{A}$. These Ext groups are defined by injective resolutions, since Grothendieck categories always have enough injectives [Ste75, Corollary X.4.3], but often do not have enough projectives. In particular, the usual characterization of a module of finite projective dimension as the 0th homology group of a finite complex of projectives will not hold in the typical Grothendieck category.

Nevertheless, objects of finite projective dimension are useful in constructing a model structure because of the following lemma.

Lemma 2.1. Suppose $\mathcal{A}$ is a Grothendieck category, $F \in \mathcal{A}$ has finite projective dimension, and $X \in \operatorname{Ch}(\mathcal{A})$ is an acyclic complex such that $\operatorname{Ext}_{\mathcal{A}}^{i}\left(F, X_{n}\right)=0$ for all $i>0$ and all $n$. Then $\mathcal{A}(F, X)$ is still acyclic.

Proof. Since $X$ is acyclic, we have a short exact sequence

$$
Z_{n} X \rightarrow X_{n} \rightarrow Z_{n-1} X .
$$

Since $\operatorname{Ext}_{\mathcal{A}}^{i}\left(F, X_{n}\right)=0$ for all $i>0$, this gives us an exact sequence

$$
0 \rightarrow \mathcal{A}\left(F, Z_{n} X\right) \rightarrow \mathcal{A}\left(F, X_{n}\right) \rightarrow \mathcal{A}\left(F, Z_{n-1} X\right) \rightarrow \operatorname{Ext}_{\mathcal{A}}^{1}\left(F, Z_{n} X\right) \rightarrow 0
$$

and isomorphisms $\operatorname{Ext}_{\mathcal{A}}^{i}\left(F, Z_{n-1} X\right) \cong \operatorname{Ext}_{\mathcal{A}}^{i+1}\left(F, Z_{n} X\right)$ for $i>0$. Thus

$$
\operatorname{Ext}_{\mathcal{A}}^{1}\left(F, Z_{n} X\right) \cong \operatorname{Ext}_{\mathcal{A}}^{m+1}\left(F, Z_{m+n} X\right)
$$

for all $m \geq 0$. Since $F$ has finite projective dimension, this implies $\operatorname{Ext}_{\mathcal{A}}^{1}\left(F, Z_{n} X\right)=$ 0 for all $n$. It follows that $\mathcal{A}(F, X)$ is acyclic.

Theorem 2.2. Suppose $\mathcal{A}$ is a Grothendieck category with a set $\mathcal{F}$ of generators, each of which has finite projective dimension. Let $\mathcal{M}$ denote the set of inclusions $A \rightarrow F$ of subobjects of objects $F \in \mathcal{F}$. Then there is a proper cofibrantly generated model structure on $\operatorname{Ch}(\mathcal{A})$, where the weak equivalences are the quasi-isomorphisms, the fibrations are the dimensionwise split surjections with dimensionwise injective kernel, and the cofibrations are the $\mathcal{M}$-cofibrations.

Note that, in a Grothendieck category, every object has a set of subobjects Ste75. Proposition IV.6.6], so $\mathcal{M}$ is really a set.

Proof. Note first that the $\mathcal{M}$-flasque objects of $\mathcal{A}$ coincide with the injective objects, by [Ste75, Prop. V.2.9]. Lemma 2.1] implies that if $X$ is an acyclic, dimensionwise injective complex, then $\mathcal{A}(F, X)$ is acyclic for all $F \in \mathcal{F}$. Hence Theorem [1.7 gives us a model structure. Any $\mathcal{M}$-fibration is a surjection with dimensionwise injective kernel, by Proposition 1.3. and therefore must be a dimensionwise split surjection. 
Conversely, a dimensionwise split surjection with dimensionwise injective kernel certainly satisfies the conditions of Proposition [1.3, so is an $\mathcal{M}$-fibration.

This model structure is related to the injective model structure; the identity functor from this model structure to the injective model structure is a Quillen equivalence. It appears to be new even when $\mathcal{A}$ is the category of modules over a ring $R$. The generating cofibrations and trivial cofibrations in this model structure are explicit, and the fibrations are easier to understand than the injective fibrations. On the other hand, we know nothing about the cofibrations in this model structure.

In general, this model structure is poorly behaved with respect to functors of abelian categories. If $F$ is an additive functor with right adjoint $U$, then $U$ will preserve fibrations in this model structure if and only if $U$ preserves injectives, which is equivalent to $F$ being exact. But this is not enough to conclude that $F$ induces a Quillen functor; we must also know that $U$ preserves acyclic complexes of injectives. This will happen if $U$ is exact, but may happen in some other cases as well.

We now consider an interesting example of this model structure. Suppose $S$ is a noetherian scheme. We say that $S$ has enough locally frees if every coherent sheaf on $S$ is a quotient of a locally free sheaf of finite rank. For example, a noetherian, integral, separated, locally factorial scheme has enough locally frees by a result of Kleiman [Har77 Ex. III.6.8].

Proposition 2.3. Suppose $S$ is a noetherian scheme with enough locally frees. In addition, suppose that $S$ is either finite-dimensional or is separated. Then the locally free sheaves of finite rank are generators of finite projective dimension for the category $\mathrm{QCo}(S)$ of quasi-coherent sheaves on $X$.

Proof. We first show that the locally frees generate $\mathrm{QCo}(S)$. Deligne Har66 Appendix, Prop. 2] has shown that every quasi-coherent sheaf is a colimit of finitely presented sheaves. On a noetherian scheme, finitely presented sheaves are coherent, and thus, since $S$ has enough locally frees, are quotients of locally free sheaves of finite rank.

Now let $F$ be a locally free sheaf of finite rank, and $C$ a quasi-coherent sheaf of $\mathcal{O}$-modules on $S$. By the corollary to Gro57, Prop. 4.2.3], we have

$$
\operatorname{Ext}_{\mathcal{O}-\text { Mod }}^{i}(F, C) \cong H^{i}(S ; \operatorname{Hom}(F, C)) \text {, }
$$

where Hom denotes sheaf Hom and the cohomology groups are sheaf cohomology. If $S$ is finite-dimensional, we can apply Grothendieck's vanishing theorem Har77. Theorem III.2.7] to conclude that these cohomology groups are 0 for large enough $i$. If $S$ is separated, then we can apply Har77, Ex. III.4.8] to reach the same conclusion, using the fact that $\operatorname{Hom}(F, C)$ is quasi-coherent.

This does not complete the proof, because these are Ext groups in $\mathcal{O}$-Mod rather than in $\mathrm{QCo}(S)$. However, these two possibly different Ext groups in fact coincide, because the exact inclusion functor $\mathrm{QCo}(S) \rightarrow \mathcal{O}$-Mod has a right adjoint and left inverse $Q$ [SGA71, p. 187] whenever $S$ is quasi-compact and quasi-separated, as any noetherian scheme is. In detail, given a quasi-coherent sheaf $C$, we can first take an injective resolution $I_{*}$ of $C$ in $\mathcal{O}$-Mod and apply $Q$ to get a complex of injectives $Q I_{*}$ in $\mathrm{QCo}(S)$. We claim that $Q I_{*}$ is still exact. To see this, consider the short exact sequence

$$
0 \rightarrow C \rightarrow I_{0} \rightarrow Z I_{1} \rightarrow 0
$$


After we apply $Q$, we get a long exact sequence involving the derived functors $R^{i} Q$ of $Q$. However, $R^{i} Q C=0$ for $i>0$, by the last paragraph of SGA71. p. 189]. Furthermore, $R^{i} Q I_{0}=0$ for $i>0$ because $I_{0}$ is injective. It follows that $\left(R^{i} Q\right) Z I_{1}=0$ for $i>0$ as well. Repeating this argument on the short exact sequence

$$
0 \rightarrow Z I_{1} \rightarrow I_{1} \rightarrow Z I_{2} \rightarrow 0
$$

we find that $\left(R^{i} Q\right) Z I_{2}=0$ for $i>0$, and, by induction, that $\left(R^{i} Q\right) Z I_{m}=0$ for all $m$ and $i>0$. Hence $Q I_{*}$ is still exact, and so is an injective resolution of $C$ in $\mathrm{QCo}(S)$.

Applying $\mathrm{QCo}(S)(B,-)$ to $Q I_{*}$ and using adjointness, we find that, if $B$ and $C$ are both quasi-coherent, then $\operatorname{Ext}_{\mathrm{QCo}(S)}^{i}(B, C)=\operatorname{Ext}_{\mathcal{O} \text {-Mod }}^{i}(B, C)$, completing the proof.

Hence, as a corollary to Proposition 2.3 and Theorem 2.2, we get the following theorem.

Theorem 2.4. Suppose $S$ is a noetherian scheme with enough locally frees, and suppose that $S$ is either finite-dimensional or separated. Then there is a proper, cofibrantly generated, model structure on the category $\mathrm{Ch}_{\mathrm{QCo}}(S)$ of unbounded complexes of quasi-coherent sheaves, where the weak equivalences are the quasi-isomorphisms and the fibrations are the dimensionwise split surjections with dimensionwise injective kernel.

Let us call this model structure the locally free model structure. We do not understand the cofibrations in the locally free model structure, though we point out that $S^{n} F$ is cofibrant for any locally free $F$, and $D^{n} A$ is cofibrant for any coherent sheaf $A$. If $f: S \rightarrow T$ is a map between schemes satisfying the hypotheses of Theorem 2.4 then the functor $f^{*}: \mathrm{QCo}(T) \rightarrow \mathrm{QCo}(S)$ will induce a Quillen functor between the locally free model structures if and only if $f^{*}$ is exact; we have already seen that this is necessary, and it is sufficient since $f^{*}$ preserves locally free sheaves of finite rank.

Despite these drawbacks, the locally free model structure does gives some information about the derived category $D(\mathrm{QCo}(S))$.

Recall that an object $A$ of a triangulated category $\mathcal{C}$ (such as $D(\mathcal{A})$ for any Grothendieck category $\mathcal{A})$ is said to be $\operatorname{small}$ if $\mathcal{C}(A,-)$ takes coproducts to direct sums (triangulated categories rarely have any other colimits). A set of weak generators for $\mathcal{C}$ is a set $\mathcal{G}$ such that $X=0$ if and only if $\mathcal{C}(G, X)_{*}=0$ for all $G \in \mathcal{G}$, where we have used the graded Hom groups always present in a triangulated category.

Corollary 2.5. Suppose $S$ is a noetherian scheme with enough locally frees, and $S$ is either finite-dimensional or separated. Then the locally free sheaves of finite rank form a set of small weak generators for the derived category $D(\mathrm{QCo}(S))$.

Proof. The fact that the locally free sheaves form a set of weak generators follows from [Hov98, Section 7.3]. To see that they are small, in the triangulated sense, we use the result of [Hov98, Section 7.4]. We must then show that, if $F$ is a locally free sheaf of finite rank, the functor $\mathrm{QCo}(S)(F,-)$ preserves all transfinite compositions. Since we are on a noetherian scheme, we can take the transfinite composition in the category of presheaves [Har77, Ex. II.1.11]. It is then easy to check the desired result. 
In case $S$ is a quasi-compact, quasi-separated scheme, we can use the right adjoint $Q$ to the inclusion $\mathrm{QCo}(S) \rightarrow \mathcal{O}$-Mod to show that $\mathrm{QCo}(S)$ is a closed symmetric monoidal category under the tensor product. Thus $\mathrm{Ch}_{\mathrm{QCo}}(S)$ is also a closed symmetric monoidal category. It would be preferable, then, to have a model structure on $\mathrm{Ch}_{\mathrm{QCo}}(S)$ that is compatible with the closed symmetric monoidal structure, in the sense of Hov98 Chapter 4]. This compatibility condition is discussed before Theorem 3.6 below. Unfortunately, the locally free model structure is not compatible with the tensor product.

Despite this, it is known that $D(\mathrm{QCo}(S))$ is a symmetric monoidal triangulated category, at least when $S$ is a finite-dimensional noetherian scheme. Indeed, Lipman [Lip98, Section 2.5] shows that $D(\mathcal{O}$-Mod) is a symmetric monoidal triangulated category. But $D(\mathrm{QCo}(S))$ is equivalent to the full subcategory of $D(\mathcal{O}$-Mod $)$ consisting of complexes with quasi-coherent cohomology, when $S$ is a finite-dimensional noetherian scheme, by SGA71, p. 191], and the inclusion $D(\mathrm{QCo}(S)) \rightarrow D(\mathcal{O}$-Mod $)$ has a right adjoint given by the right derived functor of $Q$. It follows from this that $D(\mathrm{QCo}(S))$ is a symmetric monoidal triangulated category.

Furthermore, locally free sheaves $F$ of finite rank are strongly dualizable in $D(\mathrm{QCo}(S))$. Recall that this means that the natural map

$$
\operatorname{Hom}(F, \mathcal{O}) \otimes X \rightarrow \operatorname{Hom}(F, X)
$$

is an isomorphism, where of course both the Hom and the tensor have to be interpreted in $D(\mathrm{QCo}(S))$, so are really derived versions. This follows from the corresponding fact in $\mathcal{O}$-Mod itself, and the fact that locally free sheaves are flat.

In the language of [HPS97, then, we have proved the following corollary.

Corollary 2.6. Suppose $S$ is a finite-dimensional noetherian scheme with enough locally frees. Then the category $D(\mathrm{QCo}(S))$ is a unital algebraic stable homotopy category, where the generators are the locally free sheaves of finite rank.

\section{The FLAT MODEL STRUCTURE ON SHEAVES}

In this section, we apply the method of Theorem 1.7 to the category $\mathcal{O}$-Mod of sheaves over a ringed space $(S, \mathcal{O})$. In this case, there is a standard set of generators; namely, the sheaves $\mathcal{O}_{U}$ for $U$ an open set of $S$. Recall that $\mathcal{O}_{U}$ is the sheafification of the presheaf that assigns $V$ to $\mathcal{O}(V)$ if $V \subseteq U$, and to 0 otherwise. The stalk of $\mathcal{O}_{U}$ at $x$ is 0 if $x \notin U$, and is $\mathcal{O}_{x}$ if $x \in U$. We have $\mathcal{O}-\operatorname{Mod}\left(\mathcal{O}_{U}, X\right) \cong X(U)$, which implies easily that the $\mathcal{O}_{U}$ form a generating set for $\mathcal{O}$-Mod.

Note that, if $V \subseteq U$, there is a natural monomorphism $\mathcal{O}_{V} \rightarrow \mathcal{O}_{U}$ corresponding to $1 \in \mathcal{O}_{U}(V)$. Thus, we take the set of monomorphisms $\mathcal{M}$ of the previous section to consist of these natural monomorphisms. One can then easily check that a sheaf $X$ is $\mathcal{M}$-flasque if and only if the restriction maps $X(U) \rightarrow X(V)$ are surjective whenever $V \subseteq U$, corresponding to the usual notion of a flasque sheaf.

To apply Theorem 1.7 we need to know that, if $X$ is an acyclic complex of flasque sheaves, then $\mathcal{O}-\operatorname{Mod}\left(\mathcal{O}_{U}, X\right)$ is also acyclic; i.e. that $X$ is acyclic as a complex of presheaves. Unfortunately, this need not always be true. Amnon Neeman has constructed a complex $X$ of injective sheaves on infinite-dimensional real projective space whose sheaf cohomology is trivial, but whose presheaf cohomology is nontrivial. The example is a bit complicated, but is closely related to the example in Hov98, Remark 2.3.18]. 
We therefore need a hypothesis on our ringed space to apply Theorem 1.7.

Definition 3.1. Define a ringed space $(S, \mathcal{O})$ to have finite global dimension if there is an integer $n>0$ such that $H^{n}(X)=0$ for all $\mathcal{O}$-modules $X$, where $H^{*}(X)$ denotes the sheaf cohomology groups of $X$. Define $(S, \mathcal{O})$ to have finite hereditary global dimension if every open ringed subspace $\left(U,\left.\mathcal{O}\right|_{U}\right)$ has finite global dimension.

We then get the following theorem.

Theorem 3.2. Suppose $(S, \mathcal{O})$ is a ringed space with finite hereditary global dimension. Then there is a cofibrantly generated proper model structure on $\mathrm{Ch}(\mathcal{O}-M o d)$, called the flat model structure, where the weak equivalences are the quasi-isomorphisms and the fibrations are the surjections with dimensionwise flasque kernel.

Proof. We apply Theorem 1.7, taking the set $\mathcal{M}$ to be the inclusions $\mathcal{O}_{V} \rightarrow \mathcal{O}_{U}$. We use Lemma 2.1. One can easily check that $\operatorname{Ext}_{\mathcal{O}}^{i}\left(\mathcal{O}_{U}, B\right)=H^{i}\left(U ;\left.B\right|_{U}\right)$; this is essentially the definition of sheaf cohomology. In particular, $S$ has finite hereditary global dimension if and only if each $\mathcal{O}_{U}$ has finite projective dimension. Also, since the restriction of a flasque sheaf is still flasque and flasque sheaves have no cohomology, $\operatorname{Ext}^{i}\left(\mathcal{O}_{U}, X_{n}\right)=0$ if $i>0$ and $X$ is a complex of flasque sheaves. So Lemma 2.1 applies, and Theorem 1.7 gives us the desired model structure.

The characterization of fibrations in Proposition 1.3 translates into surjections of presheaves with dimensionwise flasque kernel. However, sheaf surjections with flasque kernel are also presheaf surjections, so we get the claimed characterization of fibrations.

The author knows of two cases when ringed spaces are guaranteed to have finite hereditary global dimension.

Proposition 3.3. Suppose $(S, \mathcal{O})$ is a ringed space.

1. If $S$ is a finite-dimensional noetherian space, then $(S, \mathcal{O})$ has finite hereditary global dimension.

2. If $S$ is a finite-dimensional locally compact topological manifold that is countable at infinity, in particular if $S$ is a finite-dimensional compact manifold, then $(S, \mathcal{O})$ has finite hereditary global dimension.

Proof. Part 1 follows from the vanishing theorem [Har77, Theorem III.2.7] of Grothendieck, since an open subspace of a finite-dimensional noetherian space is still a finite-dimensional noetherian space. Part 2 is an immediate consequence of KS90. Proposition 3.2.2].

We now discuss the cofibrations in the flat model structure. Recall that the category $\mathcal{O}$-Mod is a closed symmetric monoidal category. The monoidal structure is given by the tensor product $X \otimes_{\mathcal{O}} Y$, which we will always denote by $X \otimes Y$. This is defined by forming the obvious presheaf tensor product, and sheafifying. On each stalk, the tensor product is the ordinary tensor product of modules. In particular, a sheaf $F$ is flat if and only if each stalk $F_{x}$ is flat as a $\mathcal{O}_{x}$-module; hence, the sheaves $\mathcal{O}_{U}$ are flat. The closed structure is given by the sheaf Hom; $\operatorname{Hom}(X, Y)(U)=\left.\mathcal{O}\right|_{U}-\operatorname{Mod}\left(\left.X\right|_{U},\left.Y\right|_{U}\right)$. These structures extend to complexes in the usual way, making $\mathrm{Ch}(\mathcal{O}$-Mod) into a closed symmetric monoidal category. This works for any symmetric monoidal additive category, as described in HPS97. Section 9.2] 
Definition 3.4. Suppose $\mathcal{A}$ is a symmetric monoidal abelian category. Define a complex $F \in \operatorname{Ch}(\mathcal{A})$ to be $D G$-flat if each $F_{n}$ is flat, and, for any acyclic complex $K$, the complex $F \otimes K$ is also acyclic.

Proposition 3.5. Suppose $(S, \mathcal{O})$ is a ringed space with finite hereditary global dimension. Then any cofibration in the flat model structure is a degreewise split monomorphism on each stalk, with DG-flat cokernel.

We do not know if the converse to this proposition holds, nor even whether every DG-flat complex is cofibrant. This latter statement would require that $\operatorname{Ext}_{\mathcal{O}}^{1}(F, X)=0$ for every flat sheaf $F$ and every flasque sheaf $X$.

Proof. The maps of $I$ are all degreewise split monomorphisms on each stalk. Every cofibration is a retract of a transfinite composition of pushouts of maps of $I$, by the small object argument [Hov98, Theorem 2.1.14]. Since retracts, transfinite compositions, and pushouts all commute with the operation of taking stalks and preserve split monomorphisms, each cofibration will be a degreewise split monomorphism on each stalk. The cokernel of a cofibration will of course be cofibrant, so to complete the proof it suffices to show that every cofibrant object is DG-flat.

Every cofibrant object $A$ is a retract of the colimit of a transfinite sequence $X_{\alpha}$, where each map $X_{\alpha} \rightarrow X_{\alpha+1}$ is a pushout of a map of $I$ and $X_{0}=0$. Since colimits commute with tensor products and homology, it suffices to show that, if $X_{\alpha}$ is DGflat, so is $X_{\alpha+1}$. On each stalk, the maps of $I$ are degreewise split monomorphisms with degreewise flat cokernel, so the same will be true of $X_{\alpha} \rightarrow X_{\alpha+1}$. Thus, if $X_{\alpha}$ is a complex of flat sheaves, so is $X_{\alpha+1}$.

Now suppose $K$ is an acyclic complex and $f$ is a map of $I$. Again, since the maps of $I$ are degreewise split monomorphisms on each stalk, the map $f \otimes K$ will still be injective. Thus the map $X_{\alpha} \otimes K \rightarrow X_{\alpha+1} \otimes K$, which is a pushout of $f \otimes K$ for some $f \in I$, will be injective. By the long exact sequence in homology, to show that $X_{\alpha+1}$ is DG-flat given that $X_{\alpha}$ is so, it suffices to show that $f \otimes K$ is a quasi-isomorphism. In case $f$ is of the form $D^{n} \mathcal{O}_{V} \rightarrow D^{n} \mathcal{O}_{U}$, both the domain and codomain of $f$ are contractible. The same will be true of $f \otimes K$, so $f \otimes K$ will be a quasi-isomorphism. In case $f$ is of the form $S^{n-1} \mathcal{O}_{U} \rightarrow D^{n} \mathcal{O}_{U}$, the codomain of $f \otimes K$ is contractible, so it suffices to show that $S^{n-1} \mathcal{O}_{U} \otimes K$ is acyclic. But, since $\mathcal{O}_{U}$ is flat, we have $H_{m}\left(S^{n-1} \mathcal{O}_{U} \otimes K\right)=H_{m-n+1}(K) \otimes \mathcal{O}_{U}$, so we are done.

In particular, it follows that cofibrations are pure monomorphisms, in the sense that, if $f$ is a cofibration and $K$ is an arbitrary complex, then $f \otimes K$ is still a monomorphism.

We now show that the flat model structure is compatible with the tensor product on $\mathrm{Ch}(\mathcal{O}$-Mod). To do this, we need to recall the definition of this compatibility. If $f: A \rightarrow B$ and $g: C \rightarrow D$ are maps in a cocomplete closed symmetric monoidal category, we denote the induced map

$$
(A \otimes D) \amalg_{A \otimes C}(B \otimes C) \rightarrow B \otimes D
$$

by $f \square g$. In case $\mathcal{C}$ is also a model category, we say that $\mathcal{C}$ is a symmetric monoidal model category if, whenever $f$ and $g$ are cofibrations, so is $f \square g$, and furthermore, if one of $f$ or $g$ is a trivial cofibration, so is $f \square g$. This is the condition needed to ensure that the homotopy category $\mathrm{Ho} \mathcal{C}$ is again closed symmetric monoidal, as explained in Hov98, Chapter 4]. (Actually one also needs a condition on the 
unit, but this condition is unnecessary when the unit is cofibrant, as it is in the flat model structure).

Theorem 3.6. Suppose $(S, \mathcal{O})$ is a ringed space with finite hereditary global dimension, and $f$ and $g$ are maps in $\mathrm{Ch}(\mathcal{O}-M o d)$.

(a) If $f$ is a flat cofibration and $g$ is a monomorphism, then $f \square g$ is a monomorphism.

(b) If $f$ and $g$ are flat cofibrations, then so is $f \square g$.

(c) If $f$ is a flat cofibration and $g$ is an injective quasi-isomorphism, then $f \square g$ is a quasi-isomorphism.

(d) If $f$ is a flat trivial cofibration and $g$ is a monomorphism, then $f \square g$ is a quasi-isomorphism.

Proof. As explained in [Hov98, Chapter 4], since monomorphisms and injective quasi-isomorphisms are closed under retracts, transfinite compositions, and pushouts, it suffices to check this theorem when the flat cofibration is in fact a map of $I$, and the flat trivial cofibration is a map of $J$. We begin with parts (a) and (d). Suppose that $g: A \rightarrow B$ is a monomorphism, and suppose $f$ is the map $D^{n} \mathcal{O}_{V} \rightarrow$ $D^{n} \mathcal{O}_{U}$. Let $P$ denote the domain of $f \square g$, and suppose $x \in S$. If $x \in V$, then the stalk of $P_{m}$ at $x$ is $\left(B_{m-n} \oplus B_{m-n+1}\right)_{x}$; if $x \in U \backslash V$, then the stalk of $P$ at $x$ is $\left(A_{m-n} \oplus A_{m-n+1}\right)_{x}$; and if $x$ is not in $U$, then the stalk of $P$ at $x$ is 0 . The stalk of the codomain of $f \square g$ at $x$ is $\left(B_{m-n} \oplus B_{m-n+1}\right)_{x}$ if $x$ is in $U$, and 0 otherwise, and the map $f \square g$ does the obvious thing on the stalks. Hence $f \square g$ is a monomorphism. Furthermore, the domain and codomain of $f$ are contractible, so the same will be true for $f \square g$. Thus $f \square g$ will be a quasi-isomorphism, completing the proof of part (d).

To complete the proof of part (a), we must show that $f \square g$ is a monomorphism, where now $f$ is the map $S^{n-1} \mathcal{O}_{U} \rightarrow D^{n} \mathcal{O}_{U}$. In this case, the stalk of the domain $P$ of $f \square g$ at a point $x$ is 0 if $x \notin U$, and otherwise is $\left(A_{m-n} \oplus B_{m-n+1}\right)_{x}$. The stalk of the codomain of $f \square g$ at $x$ is 0 if $x \notin U$, and otherwise is $\left(B_{m-n} \oplus B_{m-n+1}\right)_{x}$. The map $f \square g$ does the obvious thing, and so is a monomorphism.

For part (c), we can assume $f$ is the map $S^{n-1} \mathcal{O}_{U} \rightarrow D^{n} \mathcal{O}_{U}$. Then the codomain of $f \square g$ is contractible, so it suffices to show that the domain $P$ of $f \square g$ has no homology. Since $g: A \rightarrow B$ is an injective quasi-isomorphism, and $\mathcal{O}_{U}$ is flat, $g \otimes S^{n-1} \mathcal{O}_{U}$ is also an injective quasi-isomorphism. Hence its pushout $A \otimes D^{n} \mathcal{O}_{U} \rightarrow$ $P$ is also an injective quasi-isomorphism. Since $D^{n} \mathcal{O}_{U}$ is contractible, it follows that $P$ has no homology.

Finally, for part (b), we can assume that both $f$ and $g$ are maps of $I$. To calculate $f \square g$ in this case, use the easily checked (on stalks) fact that $\mathcal{O}_{U} \otimes \mathcal{O}_{V} \cong \mathcal{O}_{U \cap V}$. It follows that

$$
S^{m} \mathcal{O}_{U} \otimes S^{n} \mathcal{O}_{V} \cong S^{m+n} \mathcal{O}_{U \cap V}
$$

and

$$
S^{m} \mathcal{O}_{U} \otimes D^{n} \mathcal{O}_{V} \cong D^{m+n} \mathcal{O}_{U \cap V}
$$

and that $D^{m} \mathcal{O}_{U} \otimes D^{n} \mathcal{O}_{V}$ is an amalgamation of $D^{m+n-1} \mathcal{O}_{U \cap V}$ and $D^{m+n} \mathcal{O}_{U \cap V}$. With these identities in hand, the proof is a calculation we leave to the reader.

Corollary 3.7. Suppose $(S, \mathcal{O})$ is a ringed space with finite hereditary global dimension. Then the flat model structure makes $\mathrm{Ch}(\mathcal{O}-M o d)$ into a symmetric monoidal model category. Furthermore, if $A$ is cofibrant, then the functor $A \otimes-$ preserves 
quasi-isomorphisms. Therefore, to calculate the derived tensor product up to isomorphism, it suffices to replace one of the factors by a cofibrant complex quasiisomorphic to it.

Proof. The fact that $\mathrm{Ch}(\mathcal{O}$-Mod $)$ is a symmetric monoidal model category is immediate from Theorem 3.6. Suppose $A$ is cofibrant. Then $A \otimes-$ preserves trivial cofibrations in any symmetric monoidal model category. So, in order to see that $A \otimes-$ preserves quasi-isomorphisms, it suffices to show that, if $p$ is a trivial fibration, then $A \otimes p$ is a quasi-isomorphism. Let $K$ denote the kernel of $p$, so that $K$ is an acyclic complex. Since cofibrant objects are degreewise flat, $A \otimes p$ is still surjective, and its kernel is $A \otimes K$. Since cofibrant objects are DG-flat, $A \otimes K$ is still acyclic, so the long exact sequence completes the proof that $A \otimes-$ preserves quasi-isomorphisms. In general, the total derived functor of the tensor product is defined by $X \otimes^{L} Y=Q X \otimes Q Y$, where $Q X$ (resp. $Q Y$ ) is a functorial cofibrant replacement for $X$ (resp. $Y$ ). But, since the map $Q X \otimes Q Y \rightarrow Q X \otimes Y$ is a quasi-isomorphism, $X \otimes^{L} Y$ is isomorphic in the derived category to $Q X \otimes Y$.

Note that Theorem 3.6 actually says not only that the flat model structure is symmetric monoidal, but also that the injective model structure is a module over the flat model structure, in the sense of [Hov98, Chapter 4].

We can also use Theorem 3.6 to conclude that the derived category of $\mathcal{O}$-modules is almost a unital algebraic stable homotopy category [HPS97.

Corollary 3.8. Suppose $(S, \mathcal{O})$ is a ringed space such that $S$ is a finite-dimensional noetherian space. Then the derived category of $\mathcal{O}$-modules is a symmetric monoidal triangulated category and $\left\{\mathcal{O}_{U}\right\}$ is a set of small weak generators.

Proof. It is well-known that the derived category of any abelian category is triangulated, but this also follows, in a stronger sense of the word triangulated, from the results of [Hov98, Chapter 7]. We have already seen that the flat model category is a symmetric monoidal model category, so the derived category is also closed symmetric monoidal in a way that is compatible with the triangulation (see Hov98. Chapter 6], with one technical point dealt with by [Hov98, Corollary 5.6.10]). Since the flat model structure is cofibrantly generated, the cofibers of the generating cofibrations form a set of weak generators [Hov98, Section 7.3]. In our case, these are the objects $S^{n} \mathcal{O}_{U}$ (the cofibers of the maps of $J$ are trivial in the derived category). Because $S$ is noetherian, the presheaf colimit of a direct system of sheaves coincides with the sheaf colimit Har77, Exercise II.1.11]. It follows from this that $\mathrm{Ch}(\mathcal{O}$-Mod $)\left(S^{n} \mathcal{O}_{U},-\right)$ commutes with direct colimits. The results of Hov98, Section 7.4] then show that $S^{n} \mathcal{O}_{U}$ is small (in the triangulated sense) in the derived category.

The derived category of $\mathcal{O}$-modules is known to be a symmetric monoidal triangulated category even without the finite hereditary global dimension assumption Lip98, Section 2.5]. This might indicate that there is some replacement for the flat model structure that works more generally, or it might indicate that model categories are simply not adequate to cope with the general case.

To show that the derived category is in fact a unital algebraic stable homotopy category, we would need to know that the generators $\mathcal{O}_{U}$ are strongly dualizable. This would mean we would need to show that the natural map

$$
R \operatorname{Hom}\left(\mathcal{O}_{U}, \mathcal{O}\right) \otimes \mathcal{O}_{V} \rightarrow R \operatorname{Hom}\left(\mathcal{O}_{U}, \mathcal{O}_{V}\right)
$$


is a quasi-isomorphism. (We don't need the derived tensor product since $\mathcal{O}_{V}$ is cofibrant, using Corollary [3.7). Unfortunately, this is false in the simplest nontrivial example. Indeed, consider sheaves of abelian groups on the Sierpinski space $S$. Recall $S$ has only two points, exactly one of which is open. Sheaves on $S$ coincide with presheaves, which in turn coincide with maps $A \rightarrow B$ of abelian groups. In this case, $\mathcal{O}$ is the identity map $\mathbb{Z} \rightarrow \mathbb{Z}$, and, taking $U$ to be the open point, $\mathcal{O}_{U}$ is the map $0 \rightarrow \mathbb{Z}$. One can then calculate to find $R \operatorname{Hom}\left(\mathcal{O}_{U}, \mathcal{O}\right)=R \operatorname{Hom}\left(\mathcal{O}_{U}, \mathcal{O}_{U}\right)$, but this equality is destroyed on tensoring the left hand side with $\mathcal{O}_{U}$. A similar counterexample works if we think of $S$ as the underlying space of Spec $\mathbb{Z}_{(p)}$.

There is an additional condition that a symmetric monoidal model category might satisfy, called the monoid axiom [SS97. This axiom guarantees that the monoids in a symmetric monoidal model category, and the modules over a given monoid, themselves form model categories. The monoid axiom asserts that every map in $K$-cof is a weak equivalence, where $K$ is the class consisting of all maps $f \otimes X$, where $f$ is a trivial cofibration and $X$ is an arbitrary object.

Theorem 3.9. Suppose $(S, \mathcal{O})$ is a ringed space with finite hereditary global dimension. The the flat model structure on $\mathrm{Ch}(\mathcal{O}-M o d)$ satisfies the monoid axiom.

Proof. Suppose $f$ is a flat trivial cofibration, and $X$ is an arbitrary object. Then $0 \rightarrow X$ is a monomorphism, so applying Theorem 3.6 shows that $f \otimes X$ is an injective quasi-isomorphism. Since injective quasi-isomorphisms are closed under pushouts and transfinite compositions in any AB5 abelian category, the theorem follows.

The following corollary follows immediately from Theorem [3.9 and [SS97].

Corollary 3.10. Suppose $(S, \mathcal{O})$ is a ringed space with finite hereditary global dimension. Then:

(a) The category of monoids in $\mathrm{Ch}(\mathcal{O}-M o d)$ is a cofibrantly generated model category, where a map of monoids is a weak equivalence or a fibration if and only if it is so in the flat model structure on $\mathrm{Ch}(\mathcal{O}-M o d)$.

(b) Given a monoid $R$ in $\mathrm{Ch}(\mathcal{O}-M o d)$, the category of $R$-modules, $R$-Mod, is a cofibrantly generated proper model category, where a map of modules is a weak equivalence or a fibration if and only if it is so in the flat model structure on $\mathrm{Ch}(\mathcal{O}-\mathrm{Mod})$.

(c) If $R$ is a commutative monoid, then $R$-Mod is a symmetric monoidal model category satisfying the monoid axiom. Furthermore, the category of algebras over $R$ is a cofibrantly generated model category, where a map of algebras is a weak equivalence or fibration if and only if it is so in the flat model structure on $\mathrm{Ch}(\mathcal{O}-\mathrm{Mod})$.

The category of monoids will certainly be right proper, but the category of monoids need not be left proper in general, as pointed out by the referee. Indeed, take $\mathcal{O}=\operatorname{Spec} \mathbb{Z}$, and let $X=Y=\mathbb{Z} / 2$, thought of as a complex of quasi-coherent sheaves concentrated in degree 0 . Let $A=B$ be the standard projective resolution of $\mathbb{Z} / 2$. Then we have a weak equivalence of monoids $f: \mathbb{Z} \oplus(A \oplus B) \rightarrow \mathbb{Z} \oplus(X \oplus Y)$, where both monoids have trivial multiplication. The map $\mathbb{Z} \rightarrow \mathbb{Z}[t]$ is easily seen to be a cofibration of monoids, and so its pushout $g: \mathbb{Z} \oplus(A \oplus B) \rightarrow(Z \oplus(A \oplus B)) * \mathbb{Z}[t]$ is also a cofibration of monoids, where $*$ denotes the coproduct in the category of monoids. But the pushout of $f$ through $g$ is not a weak equivalence, because there 
will be a summand $A \otimes B$ mapping to the corresponding summand $X \otimes Y$, and the map $A \otimes B \rightarrow X \otimes Y$ is not a weak equivalence.

A map of monoids $R \rightarrow R^{\prime}$ will induce the usual induction and restriction adjunction $R$-Mod $\rightarrow R^{\prime}$-Mod. This adjunction will be a Quillen adjunction, but we would like something more.

Proposition 3.11. Suppose $(S, \mathcal{O})$ is a ringed space with finite hereditary global dimension, and $R \rightarrow R^{\prime}$ is a weak equivalence of monoids in $\mathrm{Ch}(\mathcal{O}$-Mod). Then the induction and restriction adjunction $R$-Mod $\rightarrow R^{\prime}$-Mod is a Quillen equivalence.

Proof. It suffices to show that, if $N$ is a cofibrant $R$-module, then $-\otimes_{R} N$ preserves weak equivalences, by [SS97]. The proof of this is very similar to the proof of the corresponding fact in Corollary 3.7 so we leave it to the reader.

We now investigate the functoriality of the flat model structure. Suppose we have a map of ringed spaces $f:\left(S, \mathcal{O}_{S}\right) \rightarrow\left(T, \mathcal{O}_{T}\right)$. Recall that this is a continuous map $f: S \rightarrow T$ together with a map of sheaves of rings $\mathcal{O}_{T} \rightarrow f_{*} \mathcal{O}_{S}$. Here, for any sheaf $X$ on $S, f_{*}(X)$ is the sheaf on $T$ defined by $f_{*}(X)(U)=X\left(f^{-1}(U)\right)$. If $X$ is an $\mathcal{O}_{S}$-module, then $f_{*} X$ is an $f_{*} \mathcal{O}_{S}$-module, and so an $\mathcal{O}_{T}$-module by restriction. The functor $f_{*}: \mathcal{O}_{S}$-Mod $\rightarrow \mathcal{O}_{T}$-Mod has a left adjoint $f^{*}$. To define this, recall that if $Y$ is a sheaf on $T, f^{-1} Y$ is the sheaf on $S$ associated to the presheaf that takes $U$ to $\operatorname{colim}_{V \supseteq f(U)} Y(V)$. The functor $f^{-1}$ is left adjoint to $f_{*}$ on the category of sheaves of abelian groups, so in particular we have a map $f^{-1} \mathcal{O}_{T} \rightarrow \mathcal{O}_{S}$. Given an $\mathcal{O}_{T}$-module $Y$, we define $f^{*} Y=\mathcal{O}_{S} \otimes_{f^{-1} \mathcal{O}_{T}} f^{-1} Y$. It is well-known that $f^{*}$ is left adjoint to $f_{*}$ and is symmetric monoidal [Gro60, Section 0.4.3].

One can verify using adjointness that, if $U$ is an open subset of $T$, then $f^{*} \mathcal{O}_{U}=$ $\mathcal{O}_{f^{-1} U}$. Hence we have the following proposition.

Proposition 3.12. Suppose $f:\left(S, \mathcal{O}_{S}\right) \rightarrow\left(T, \mathcal{O}_{T}\right)$ is a map of ringed spaces with finite hereditary global dimension. Then $f^{*}$ is a left Quillen functor with respect to the flat model structures.

In particular, this shows that the total left derived functor of $f^{*}$ exists and is left adjoint to the total right derived functor of $f_{*}$. It is proved in Lip98 Section 2.7] that the total left derived functor of $f^{*}$ exists without the finite hereditary global dimension hypotheses. It is disconcerting that we are unable to reproduce this result using model categories.

\section{Appendix A. Smallness}

The object of this appendix is to prove that every object in a Grothendieck category is small. This follows immediately from the fact that Grothendieck abelian categories are precisely the locally presentable abelian categories Bek99. Proposition 3.10]. Beke's proof, and the proof of smallness we give here, depend crucially on the Gabriel-Popescu theorem [Ste75, Theorem X.4.1], which shows that every Grothendieck abelian category is the localization of a module category with respect to a hereditary torsion theory. The proof in Beke relies on general theorems about locally presentable categories, whereas the proof we give uses properties of the localization functor (which can be found in [Ste75]).

We begin with a precise definition of the term "small". 
Definition A.1. 1. Given a limit ordinal $\lambda$, the cofinality of $\lambda$, cofin $\lambda$, is the smallest cardinal $\kappa$ such that there exists a subset $T$ of $\lambda$ with $|T|=\kappa$ and $\sup T=\lambda$.

2. Given an object $A$ in a cocomplete category $\mathcal{C}$ and a cardinal $\kappa$, we say that $A$ is $\kappa$-small if, for every ordinal $\lambda$ with cofin $\lambda>\kappa$ and every colimit-preserving functor $X: \lambda \rightarrow \mathcal{C}$, the natural map $\operatorname{colim}_{i<\lambda} \mathcal{C}\left(A, X_{i}\right) \rightarrow \mathcal{C}\left(A, \operatorname{colim}_{i<\lambda} X_{i}\right)$ is an isomorphism.

3. An object $A$ in a cocomplete category $\mathcal{C}$ is called small if it is $\kappa$-small for some cardinal $\kappa$.

Proposition A.2. Every object in a Grothendieck category is small.

Proof. By the Gabriel-Popescu theorem [Ste75, Theorem X.4.1], we may as well assume that our Grothendieck category $\mathcal{A}$ is the localization of $R$-Mod with respect to a hereditary torsion theory $\mathcal{T}$, for some ring $R$. Let $\kappa$ be the larger of $\omega$ and the cardinality of $R$, let $\lambda$ be an ordinal with cofin $\lambda>\kappa$, and let $X: \lambda \rightarrow \mathcal{A}$ be a colimit-preserving functor. We will first show that $\operatorname{colim} X_{i}$, calculated in $R$-Mod, is still $\mathcal{T}$-local, so is also the colimit in $\mathcal{A}$. This proof will depend on the fact that both $R / \mathfrak{a}$ and $\mathfrak{a}$ are $\kappa$-small in $R$-Mod [Hov98, Example 2.1.6], for all (left) ideals $\mathfrak{a}$ of $R$.

To see this, first note that colim $X_{i}$ is torsion-free. Indeed, $\mathcal{T}$ is generated by cyclic modules $R / \mathfrak{a}$, so it suffices to show that $R-\operatorname{Mod}\left(R / \mathfrak{a}, \operatorname{colim} X_{i}\right)=0$. But we have chosen $\kappa$ so that

$$
R-\operatorname{Mod}\left(R / \mathfrak{a}, \operatorname{colim} X_{i}\right) \cong \operatorname{colim} R-\operatorname{Mod}\left(R / \mathfrak{a}, X_{i}\right),
$$

and this is 0 since each $X_{i}$ is torsion-free. Hence colim $X_{i}$ is torsion-free.

It follows that the localization of $\operatorname{colim} X_{i}$ is

$$
\operatorname{colim}_{\mathfrak{a}} \operatorname{Hom}\left(\mathfrak{a}, \operatorname{colim}_{i} X_{i}\right),
$$

where the colimit is taken over ideals $\mathfrak{a}$ such that $R / \mathfrak{a}$ is torsion, as in Ste75. Section IX.1]. But then we have

$$
\begin{aligned}
\operatorname{colim}_{\mathfrak{a}} \operatorname{Hom}\left(\mathfrak{a}, \operatorname{colim}_{i} X_{i}\right) & \cong \operatorname{colim}_{\mathfrak{a}} \operatorname{colim}_{i} \operatorname{Hom}\left(\mathfrak{a}, X_{i}\right) \\
& \cong \operatorname{colim}_{i} \operatorname{colim}_{\mathfrak{a}} \operatorname{Hom}\left(\mathfrak{a}, X_{i}\right)=\operatorname{colim}_{i} X_{i} .
\end{aligned}
$$

Thus colim $X_{i}$ is already local.

Now suppose $M$ is an arbitrary local module. There is a cardinal $\kappa^{\prime}$ such that $M$ is $\kappa^{\prime}$-small as an $R$-module, and we can choose $\kappa^{\prime} \geq \kappa$. It is then immediate from the argument above that $M$ is $\kappa^{\prime}$-small in $\mathcal{A}$.

\section{REFERENCES}

[Bek99] Tibor Beke, Sheafifiable homotopy model categories, preprint, 1999.

[Chr98] J. Daniel Christensen, Derived categories and projective classes, preprint, 1998.

[DS95] W. G. Dwyer and J. Spalinski, Homotopy theories and model categories, Handbook of Algebraic Topology, North-Holland, Amsterdam, 1995, pp. 73-126. MR 96h:55014

[Gro57] Alexander Grothendieck, Sur quelques points d'algèbre homologique, Tôhoku Math. J. (2) 9 (1957), 119-221. MR 21:1328

[Gro60] A. Grothendieck, Éléments de géométrie algébrique. I. Le langage des schémas, Inst. Hautes Études Sci. Publ. Math. No. 4 (1960). MR 36:177a

[Har66] R. Hartshorne, Residues and duality, Lecture Notes in Mathematics, vol. 20, SpringerVerlag, 1966. MR 36:5145

[Har77] Robin H. Hartshorne, Algebraic geometry, Graduate Texts in Mathematics, vol. 52, Springer-Verlag, 1977. MR 57:3116 
[Hov98] Mark Hovey, Model categories, Mathematical Surveys and Monographs, vol. 63, American Mathematical Society, Providence, RI, 1998. MR 99h:55031

[HPS97] Mark Hovey, John H. Palmieri, and Neil P. Strickland, Axiomatic stable homotopy theory, Mem. Amer. Math. Soc. 128 (1997), no. 610. MR 98a:55017

[Joy84] A. Joyal, Letter to A. Grothendieck, 1984.

[KS90] Masaki Kashiwara and Pierre Schapira, Sheaves on manifolds, Springer-Verlag, Berlin, 1990, With a chapter in French by Christian Houzel. MR 92a:58132

[Lip98] Joseph Lipman, Notes on derived categories and derived functors, preprint, 1998.

[MP92] I. Moerdijk and D. A. Pronk, Cohomology of sheaves, lecture notes, 1992.

[Qui67] Daniel G. Quillen, Homotopical algebra, Lecture Notes in Mathematics, vol. 43, SpringerVerlag, 1967. MR 36:6480

[SGA71] Théorie des intersections et théorème de Riemann-Roch, Springer-Verlag, Berlin, 1971, Séminaire de Géométrie Algégrique du Bois-Marie 1966-1967 (SGA 6), Dirigé par P. Berthelot, A. Grothendieck et L. Illusie. Avec la collaboration de D. Ferrand, J. P. Jouanolou, O. Jussila, S. Kleiman, M. Raynaud et J. P. Serre, Lecture Notes in Mathematics, Vol. 225, Springer-Verlag, 1971. MR 50:7133

[Spa88] N. Spaltenstein, Resolutions of unbounded complexes, Compositio Math. 65 (1988), no. 2, 121-154. MR 89m:18013

[SS97] Stefan Schwede and Brooke Shipley, Algebras and modules in monoidal model categories, Proc. London Math. Soc. (3) 80 (2000), 491-511. CMP 2000:07

[Ste75] B. Stenström, Rings of quotients, Die Grundlehren der mathematischen Wissenschaften, vol. 217, Springer-Verlag, Berlin, 1975. MR 52:10782

[TLS99] L. Alonso Tarrío, A. Jeremias López, and M. J. Souto Salorio, Localizations in categories of complexes and unbounded resolutions, Canad. J. Math. 52 (2000), 225-247. CMP 2000:12

Department of Mathematics, Wesleyan University, Middletown, Connecticut 06459

E-mail address: hovey@member.ams.org 